\title{
A single vectored thermal pulsation treatment for meibomian gland dysfunction increases mean comfortable contact lens wearing time by approximately 4 hours per day
}

This article was published in the following Dove Press journal: Clinical Ophthalmology

\author{
Caroline A Blackie' \\ Christy A Coleman' \\ Kelly K Nichols ${ }^{2}$ \\ Lyndon Jones ${ }^{3}$ \\ Peter Q Chen ${ }^{4}$ \\ Ron Melton ${ }^{5}$ \\ David L Kading ${ }^{6}$ \\ Leslie E O’Dell ${ }^{7}$ \\ Sruthi Srinivasan ${ }^{3}$ \\ 'TearScience Inc., Morrisville, NC, \\ ${ }^{2}$ School of Optometry, The University \\ of Alabama at Birmingham, AB, USA; \\ ${ }^{3}$ School of Optometry and Vision \\ Science, University of Waterloo, \\ Waterloo, ON, Canada; ${ }^{4}$ Clayton \\ Eye Center, Morrow, GA, ${ }^{5}$ Charlotte \\ Eye Ear Nose and Throat Associates, \\ Charlotte, NC, ${ }^{6}$ Specialty Eyecare \\ Group, Kirkland, WA, ${ }^{7}$ May Eye Care \\ Center and Associates, Hanover, \\ PA, USA
}

\begin{abstract}
Purpose: To evaluate the effect of a single vectored thermal pulsation (VTP) treatment in contact lens wearers with meibomian gland dysfunction (MGD) and dry eye symptoms.

Methods: The prospective, nonsignificant risk, open-label, randomized, multi-center clinical trial included 55 soft contact lens (SCL) wearers with MGD and evaporative dry eye. Subjects were randomized to the single VTP treatment group or an untreated control. The controls received a crossover VTP treatment at 3 months (crossover treatment group). Primary effectiveness measures were meibomian gland secretion (MGS) score and Standard Patient Evaluation of Eye Dryness (SPEED) that were evaluated at baseline, at 1 and 3 months post-VTP treatment, and at 1 month post-VTP treatment in the crossover treatment group. Exploratory variables included fluorescein tear break-up time (TBUT), lid wiper epitheliopathy (LWE), lid parallel conjunctival folds (LIPCOF), ocular surface staining, frequency of over-the-counter (OTC) drop use, and hours of comfortable contact lens wear.
\end{abstract}

Results: At 3 months, the treatment group showed significantly greater mean change from baseline in MGS (12.4 \pm 9.1 vs $1.4 \pm 6.4, \mathrm{p}<0.0001)$, SPEED $(-8.4 \pm 4.7$ vs $-0.7 \pm 4.4, \mathrm{p}<0.0001)$ and significantly greater improvement in exploratory variables (TBUT, LWE, and frequency of OTC drop use) relative to the controls. Mean comfortable contact lens wearing time increased by $4.0 \pm 3.9$ hours at 1 month. This was sustained for 3 months with no change in the control group. The crossover treatment group demonstrated similar results to the treatment group at 1 month post-VTP.

Conclusion: In SCL wearers with MGD, a single VTP treatment significantly improved mean meibomian gland function and significantly reduced dry eye signs and symptoms compared to an untreated control. The treatment increased mean comfortable lens wearing time by 4 hours (approximately doubling the pretreatment findings). This was sustained for up to 3 months post-treatment on average.

Keywords: meibomian gland dysfunction, vectored thermal pulsation, Dynamic Meibomian Imaging, evaporative dry eye, soft contact lens wear, comfortable contact lens wearing time

\section{Introduction}

Symptoms of dryness and discomfort are highly prevalent (up to 50\%) among contact lens wearers and are the most commonly cited reason for the discontinuation of contact lens wear. ${ }^{1-4}$ Despite decades of advances in contact lens design and materials used to manufacture the lenses, the contact lens dropout rate remains consistently high at $16 \%-34 \%$ per year. ${ }^{4,5}$ The improvements in contact lens design serve, in part,
Correspondence: Caroline A Blackie TearScience Inc., 5I5I McCrimmon Parkway, Suite 250, Morrisville, NC 27560, USA

Tel + I 9194594880

Fax + I 9194673300

Email cblackie@its.jnj.com (c) (1) (5) 2018 Blackie et al. This work is published and licensed by Dove Medical Press Limited. The full terms of this license are available at https://www.dovepress.com/terms.php cc) hereby accept the Terms. Non-commercial uses of the work are permitted without any further permission from Dove Medical Press Limited, provided the work is properly attributed. For permission for commercial use of this work, please see paragraphs 4.2 and 5 of our Terms (https://www.dovepress.com/terms.php). 
to minimize the discomfort caused by the physical changes, which occur to a contact lens within minutes and hours of placement on the eye (eg, lens dehydration, pre-lens tear film stagnation, and post-lens debris). ${ }^{6-8}$ However, regardless of lens design optimization, there are ocular tissue changes that occur due to the disruptive presence of any contact lens on the eye. ${ }^{3,6-8}$ Examples of ocular tissue changes that have been reported as strongly correlated with contact lens wear are lid wiper epitheliopathy (LWE), lid parallel conjunctival folds (LIPCOF), and meibomian gland dysfunction (MGD). ${ }^{9-11}$ The mechanisms responsible for these tissue changes can be largely categorized as the result of increased evaporative stress on the tear film, friction between the lid wiper and the ocular surface, and the resulting inflammatory cascades that predictably ensue. ${ }^{12}$ Generally speaking, even with optimized contact lens design and materials, the importance of a stable and robust tear film and homeostatic ocular surface environment cannot be overstated, if successful contact lens wear is to be achieved. ${ }^{6,13,14}$

While the volume of literature regarding the need for tear film stability in order to achieve comfortable contact lens wear is significant, the full extent of the impact of a contact lens on the ocular surface is not fully understood or characterized. A recent publication hypothesizes the adverse effect of a contact lens on the eye through the mechanism of chronic desiccating stress. ${ }^{15}$ Chronic exposure to desiccating stress has been shown to result in protracted overstimulation of mouse meibomian gland meiboctyes. The unrelenting meibocyte upregulation accelerated the aging of the meibocytes, altered gland secretion quality, and ultimately led to gland atrophy in what were once healthy meibomian glands. ${ }^{15}$ While the direct link between MGD and chronic exposure to desiccating stress, alone or in contact lens wear, has not been demonstrated in the human eye, the reported association between contact lens wear and MGD, now spanning several decades, is well established. ${ }^{11,16,18-20}$

The multiple studies noting the high prevalence and increased severity of MGD in contact lens wearers ${ }^{11,16,18-20}$ significantly outnumber reports to the contrary. ${ }^{21}$ Regardless, even if no causal relationship between contact lens wear and MGD has been demonstrated, MGD will have a predictable negative impact on contact lens wear over both the short and long terms. ${ }^{18,19}$ In addition, any contact lens wearer with MGD is at risk for ocular discomfort and dry eye due to the MGD and evaporative dry eye. ${ }^{19}$ Recently, MGD has been shown to negatively impact tear film host defense, ${ }^{22}$ corneal epithelial cell and stromal nerve health, ${ }^{23}$ conjunctival health, ${ }^{24}$ and various other measures of ocular surface health. ${ }^{25}$
These studies indicate that MGD compromises ocular surface health in multiple, foreseeable, and measurable ways, none of which would be advantageous for contact lens wear.

Previous studies have shown that when MGD is treated, contact lens wear is positively impacted in the form of improved tear film stability, increased comfortable contact lens wearing time, and reduced LWE. ${ }^{16,17,26}$ The expanding body of evidence demonstrating the negative impact of MGD on ocular surface health in general and on contact lens wear, in particular, has led others to recommend that contact lens wearers should be routinely evaluated and treated for MGD. ${ }^{18}$

Vectored thermal pulsation (VTP) treatment (LipiFlow ${ }^{\circledR}$; TearScience Inc., Morrisville, NC, USA) is designed to directly treat MGD by evacuating the contents of both the upper and lower meibomian glands simultaneously during a single treatment. ${ }^{27}$ While the success of the LipiFlow in treating MGD has been reported extensively, ${ }^{27-33}$ there is only one report of a small pilot study treating contact lens wearers with MGD with the LipiFlow System. ${ }^{26}$ The results of this pilot study showed that a single VTP treatment increased mean daily contact lens wearing time by 3.6 hours as well as significantly reducing LWE. However, the study had several limitations, such as being a retrospective study at a single site. The purpose of this study was to prospectively assess the effect of a single VTP treatment in contact lens wearers with MGD on contact lens-related dry eye signs and symptoms in a randomized, multi-center clinical trial.

\section{Methods}

This prospective, multi-center clinical trial was conducted in compliance with US Code of Federal Regulations (CFR): 21 CFR Parts 50, 54, 56, and 812 and the Canadian Medical Device Regulations. The study was performed under the approval of three Institutional Review Boards (Schulman Associates IRB, Cincinnati, OH, USA; Western IRB, Puyallup, WA, USA; and University of Waterloo Office of Research Ethics, Waterloo, ON, Canada), and all tenets of the Declaration of Helsinki for the protection of human subjects in medical research were strictly observed (including obtaining written informed consent from all patients). The study involved postmarket use of the LipiFlow System and commercially available contact lenses. A sub-study involved the investigational use of the TearScience Meibographer as a nonsignificant risk device study at US sites only. This study was registered at the US National Institutes of Health (ClinicalTrials.gov; \#NCT02102464). Between May 20, 2014, and February 5, 2015, 55 adult subjects with MGD 
and dry eye symptoms were randomized in the study at six sites in the USA and Canada. Subjects were not required to pay for their designated treatment.

\section{Study design}

This study was an open-label, randomized, multi-center, clinical trial of a single VTP treatment vs an untreated control in contact lens wearers with MGD and evaporative dry eye. Subjects were randomized to receive a single VTP treatment in both eyes (treatment group) or no VTP treatment (untreated control group). Study endpoints were evaluated at 3 months by comparing the mean change from baseline to 3 months for treatment group with the untreated group. To facilitate subject recruitment, the untreated control group received a crossover VTP treatment (crossover treatment group) at 3 months. Both the treatment and crossover treatment groups were evaluated 1 month after receiving VTP treatment (Figure 1).

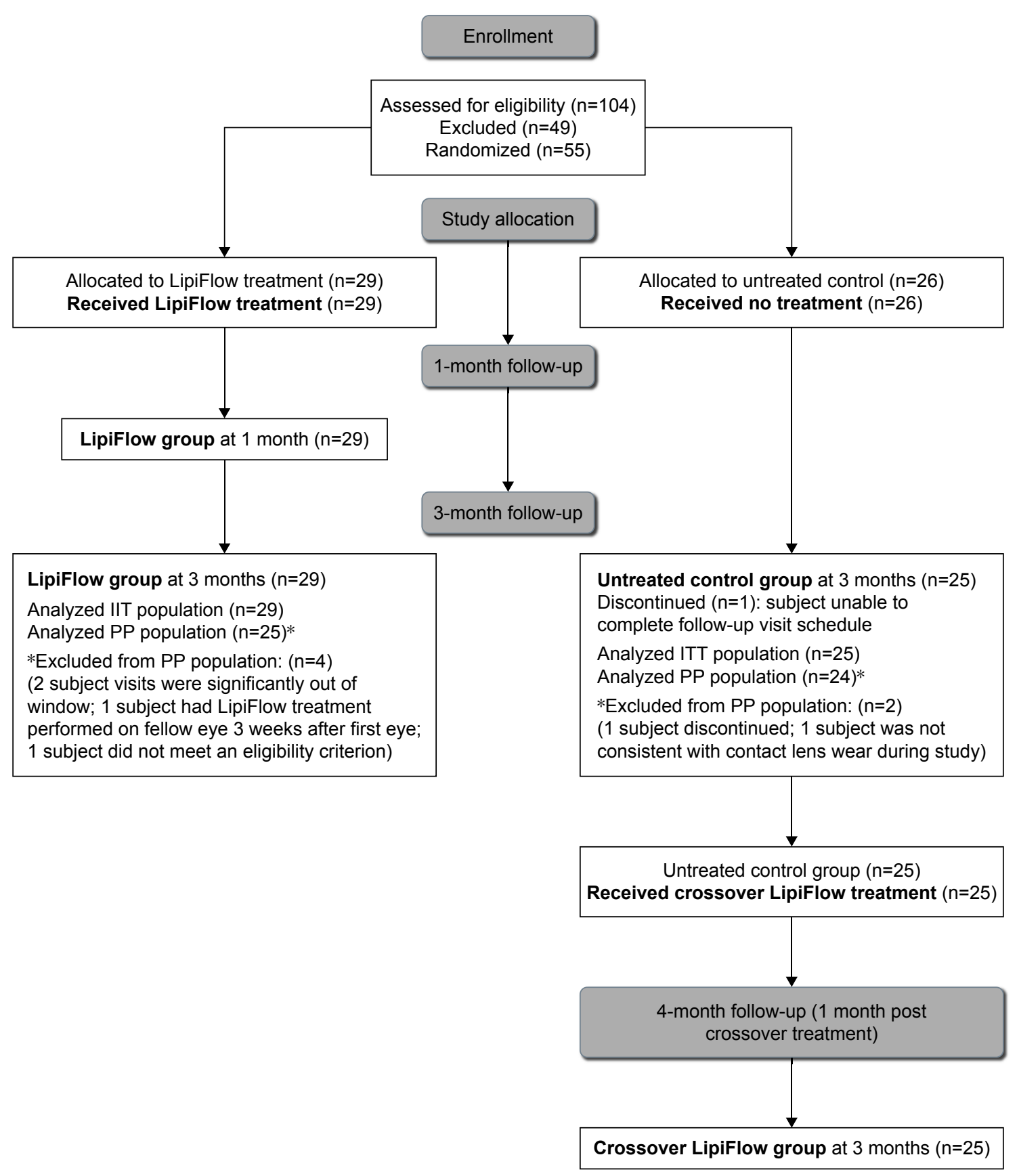

Figure I An abbreviated CONSORT flow diagram indicating the complete subject disposition throughout the trial. Abbreviations: ITT, intent-to-treat; PP, per protocol. 


\section{Inclusion criteria}

Patients were required to be aged at least 18 years and willing and able to comply with the study protocol, have MGD (a meibomian gland secretion [MGS] score of $\leq 15$ for 15 glands of the lower lid) in both eyes; have a Standard Patient Evaluation of Eye Dryness (SPEED) questionnaire score of $\geq 6 ;{ }^{34}$ have a minimum lipid layer thickness of $\leq 100 \mathrm{~nm}$ in both eyes (LipiView); have a desire to wear contact lenses habitually and not for intentional occasional or recreational wear only; wear soft daily contact lenses in both eyes with a total wearing time of 2-18 hours a day and 4-7 days a week on average (the original protocol, Protocol Revision 1, included a range of 2-12 hours of wearing time; this was increased in Protocol Revision, 2-18 hours, to facilitate recruitment); classified as having dry eye while wearing contact lenses based on composite score on Contact Lens Dry Eye Questionnaire (CLDEQ) - Long Form; ${ }^{35}$ have worn the same type of commercially available soft contact lens (SCL) (brand, material, and dimensions) for at least the past 3 months (the original protocol, Protocol Revision 1, stated 6 months, which was reduced to 3 months in Protocol Revision 2 to facilitate recruitment); and have an assessment based on the clinician's experience and judgment that contact lens fit, lens material, and compatible disinfecting solution were acceptable and not the reasons for contact lens discomfort. An additional criterion in Protocol Revision 1 was a comfortable contact lens wearing time of $\leq 4$ hours per day on average; this criterion was removed in Protocol Revision 2 to facilitate recruitment.

\section{Exclusion criteria}

Conditions that could potentially interfere with the evaluation of or compromise treatment effectiveness or increase the risk of a procedure-related injury were listed as exclusion criteria. These conditions included moderate-to-severe allergic, vernal, or giant papillary conjunctivitis; active ocular infection or inflammation; ocular surface abnormalities compromising corneal integrity; eyelid abnormalities that could compromise lid function; recent (within the past 3 months) ocular surgery, herpes, or recurrent inflammation; systemic disease associated with dry eye; systemic medications known to cause dryness; current use of prescription medications and other treatments for MGD or dry eye (excluding over-the-counter [OTC] artificial tears, dietary supplements, and ocular lubricants); unwillingness to discontinue the use of these medications/treatments for the study duration (Protocol Revision 1 excluded the current use of these medications and treatments for the past
90 days, which was reduced to the past 30 days in Protocol Revision 2 to facilitate recruitment); and participation in another ophthalmic clinical trial involving a device or therapeutic drug within the past 30 days and employee, associate, or relative of an employee at a clinical study site.

\section{Randomization and subject disposition}

A total of 29 subjects (58 eyes) were randomized to the treatment group and 26 subjects (52 eyes) to the untreated control group. The control group received no treatment, while the treatment group received a single 12-minute VTP treatment with the LipiFlow System. The treatment group received manual eyelid margin cleaning prior to the VTP treatment ${ }^{36}$ and were instructed to perform blinking exercises (Figure S1) for 1 month after the treatment visit to foster healthy blinking habits. ${ }^{37}$

Subjects in the control group were evaluated at 3 months post-baseline visit. After the examination at the 3 months post-baseline visit, the control subjects received a single 12-minute crossover VTP treatment and were evaluated again 1 month post-VTP treatment. Subjects in the treatment group were evaluated at 1 and 3 months post-VTP treatment. The complete subject disposition is given in the form of an abbreviated CONSORT flow diagram in Figure 1.

\section{The LipiFlow System}

The LipiFlow System has been described in detail elsewhere. ${ }^{27}$ Briefly, it is a prescription device for in-office use by a physician. The LipiFlow System is indicated the treatment of MGD and lipid deficiency or evaporative dry eye. The device applies controlled therapeutic heat to the inner eyelid surface with simultaneous intermittent directional pressure to the outer eyelid to facilitate evacuation of the gland contents during the heating phase of the treatment. ${ }^{27}$

\section{Study parameters}

Study endpoint parameters were meibomian gland assessment and the SPEED questionnaire. These parameters were assessed at all study visits except the treatment visit. Meibomian gland assessment was performed using a previously described handheld instrument, Meibomian Gland Evaluator (MGE), to apply standardized pressure to the eyelid margin, which approximates the force of a deliberate blink over the glands. ${ }^{38} \mathrm{~A}$ total of 15 glands were evaluated along the lower eyelid margin. This is achieved by evaluating five glands in each of the temporal, central, and nasal regions of the eyelid. Gland secretion characteristics were graded as 3 (clear liquid secretion), 2 (cloudy liquid secretion), 
1 (inspissated/toothpaste consistency), and 0 (no secretion). The total MGS score for each eye was calculated based on the sum of the secretion grades (range of $0-45$ ) for all evaluated glands. ${ }^{38}$ In addition, the total number of functional meibomian glands, defined as having liquid secretion with a grade of 2 or 3, was counted with a range of $0-15 .{ }^{27}$

The frequency and severity of dry eye symptoms were assessed with the SPEED questionnaire. The total SPEED score (range from 0 to 28) was calculated as the sum of frequency and severity scores for all questions answered. ${ }^{34}$

\section{Dynamic Meibomian Imaging (DMI)}

Meibomian gland structure was captured on a subset of subjects ( $n=37$, US sites only) using an investigational device (TearScience Meibographer; TearScience Inc.) with DMI. Meibomian gland structure was imaged at all study visits except the treatment visit. After data collection, an independent reader graded the gland structure according to a previously accepted method where the amount of atrophy in each eyelid (upper and lower) is semiquantitatively assessed and recorded as Grades 0-3: Grade 0, no atrophy; Grade 1, 1\%-33\% atrophy; Grade 2, 34\%-66\% atrophy; Grade 3, $67 \%-100 \%$ atrophy. ${ }^{39-41}$ The upper and lower eyelid atrophy scores were then combined to form a total combined meiboscore for each eye (maximum score $=6$ ) and an average eyelid meiboscore (maximum score $=3$ ). ${ }^{39-41}$

\section{Tear break-up time (TBUT)}

The exploratory endpoint, TBUT, was measured at all study visits except the treatment visit. The dry eye test (DET) (Amcon Laboratories, St Louis, MO, USA) was used to instill fluorescein dye. ${ }^{42}$ Three separate measurements of TBUT were taken for each eye using a stopwatch to record the time. If breakup was not observed within 20 seconds, the measurement was to be terminated to prevent possible corneal drying. For data analysis, the three measurements were averaged to represent the mean TBUT for each eye.

\section{Ocular surface staining}

At all study visits except the treatment visit, corneal staining and conjunctival staining were evaluated with fluorescein and lissamine green, respectively, and recorded using standard methods on a scale of $0-3$ reported in Lemp et al. ${ }^{43}$ The total staining grade for the cornea was the sum of the grades for all five corneal regions (0-15). The total staining grade for the conjunctiva was the sum of the grades for all six conjunctival regions $(0-18)$. The respective dyes were instilled using commercially available saline and standard fluorescein
(Akorn, Inc., Farmington, CT, USA) and lissamine green strips (Bernell Corporation, Mishawaka, IN, USA). Staining was evaluated 90 seconds after the instillation of each respective dye. The corneal staining with fluorescein was completed and recorded prior to the instillation of lissamine green for the conjunctival staining procedure.

\section{LWE}

The full length and width of the wiper were examined for staining and graded using both standard fluorescein and lissamine green dye ${ }^{9}$ at all study visits except the treatment visit. Both dyes were instilled at the same time, twice, 90 seconds apart, using commercially available saline and standard fluorescein and lissamine green strips. Staining was evaluated 90 seconds after the instillation of the second combined dye instillation. Staining of the horizontal length was graded on a scale of 0 ( $<2 \mathrm{~mm}), 1(2-4 \mathrm{~mm}), 2(5-9 \mathrm{~mm})$ and $3(\geq 10 \mathrm{~mm})$. Staining of the sagittal height was graded on a scale of $0(<25 \%), 1$ (25 to $<50 \%), 2$ (50 to $<75 \%)$, and $3(\geq 75 \%) .{ }^{9}$ For data analysis, individual grades for length and width were averaged for a final grade for each staining method. The higher of the final fluorescein or lissamine green staining grades was used as the LWE severity grade.

\section{LIPCOF}

At all study visits except the treatment visit, the area perpendicular to the temporal and nasal limbus on the bulbar conjunctiva above the lower lid was assessed for LIPCOF under $18-24 \times$ slit lamp biomicroscope magnification and graded on a scale of $0-3$ according to a previously accepted method: ${ }^{10}$ Grade 0, no conjunctival folds visible; Grade 1, one permanent and clear parallel conjunctival fold $(\sim 0.05 \mathrm{~mm}$ thick or thicker); Grade 2, two permanent and clear parallel conjunctival folds; Grade 3, more than two permanent and clear parallel conjunctival folds. ${ }^{10}$

\section{Frequency of OTC drop use}

The study permitted concomitant use of pre-existing OTC medications for MGD or dry eye, including artificial tears, ocular lubricants, and dietary supplements. However, subjects were instructed not to start any new OTC medications for the study duration. To control the concomitant use, subjects were required to report on the dosage and frequency of use at the initial visit to document the pre-existing OTC medications and, at each subsequent visit, to assess for any change in use during the study. To quantify the change in usage over time for those subjects taking topical OTC medications, 
the monthly frequency of topical OTC use was calculated assuming 30 days and 4 weeks in a month.

\section{Total and comfortable contact lens wearing time}

Total contact lens wearing time and comfortable contact lens wearing time in hours per day were reported by each subject at all study visits except the treatment visit. The information was captured in response to the following questions: 1) How many hours a day does the subject wear SCLs on average? And 2) How many hours a day does the subject report comfortable contact lens wear on average? ${ }^{44,45}$

\section{Other questionnaire metrics}

In addition to the SPEED questionnaire, subjects were selfadministered the CLDEQ - Long Form ${ }^{36,46}$ - and Ocular Surface Disease Index (OSDI) questionnaire. ${ }^{47,48}$ CLDEQ was used to determine study eligibility based on having a diagnosis of dry eye while wearing contact lenses and to evaluate contact lens comfort related to dry eye. The OSDI questionnaire assessed the subjects' frequency of dry eye symptoms in specific contexts. Total OSDI score was calculated as the sum of frequency scores for all symptoms multiplied by 25 and divided by the number of questions answered with a range from 0 to 100 . Sub-scores for ocular symptoms, vision-related functioning, and environmental conditions were similarly calculated. ${ }^{47}$

As a part of the questionnaire following VTP treatment or crossover VTP treatment, subjects were asked to report if the treatment improved their overall dry eye symptoms and, if yes, the percentage improvement on a scale from 10 to 100 in $10 \%$ increments. In addition, subjects were asked to report if they were able to perform any task better or longer as a result of treatment and, if yes, to list the task(s).

\section{Study endpoints}

The primary and secondary endpoints were the mean change in MGS and SPEED scores, respectively, from baseline to 3 months between the treated and untreated control groups. The primary study endpoint was used in the power analysis to determine study sample size. Adverse event data were collected at all study visits, but there were no safety endpoints in this study. The LipiFlow System is a nonsignificant risk device, and safety has been previously demonstrated. ${ }^{27}$

\section{Additional exploratory analyses}

Additional exploratory analyses included TBUT, analysis of meibography images, and comparison between the treatment and untreated control groups in the mean change from baseline to 3 months in TBUT, LWE, LIPCOF, frequency of OTC drop use, total contact lens wearing time, and comfortable contact lens wearing time.

\section{Statistical analysis}

Statistical analysis was performed using the SAS (SAS Institute Inc., Cary, NC, USA) software Version 9.4. A twosample $t$-test (one-sided alpha 0.025 ) was used to test the primary and secondary endpoints for the change from baseline to 3 months in meibomian gland score and SPEED score, respectively, between the treatment and untreated control groups. Descriptive statistics was provided for age, gender, race, ethnicity baseline measurements, and study examination findings summarized by group.

A comparability analysis was done by group and by protocol revisions ( 1 and 2 ) to assess the poolability of the data for demographic and baseline measurements. For quantitative variables, the comparison was done using twosample $t$-tests or Wilcoxon rank sum tests, as appropriate. For qualitative variables, a Fisher's exact test was used. Contact lens wear analyses were stratified by protocol revision and group because of the difference in inclusion criteria for total and comfortable contact lens wearing times between protocol revisions and to control for associated statistically significant baseline differences in wearing times.

\section{Subject instructions and compliance}

Subjects were provided with written instructions for contact lens wear, medication use during the study, and blinking exercises. Subject compliance with these instructions was reviewed at follow-up visits. At each visit, the subjects were reminded not to change their brand or type of contact lenses or disinfecting solution during the study period unless so advised by their eye doctor and to report any changes at subsequent visits as needed.

The medication instructions included a subject reminder to abstain from using any prescribed medication that can cause dry eye or prescribed medication or treatment intended to treat dry eye or MGD. Except for medical management of an adverse event, investigators were not permitted to prescribe or administer any new treatments for dry eye or MGD (including prescription medications, warm compresses, eyelid warming, eyelid massage, eyelid hygiene, meibomian gland expression, meibomian gland probing, punctal plug insertion, punctal occlusion, intense pulse light treatment of the face or eyelids, and additional LipiFlow treatment) for the study duration. For those subjects who were using 
pre-existing OTC products (eg, contact lens wetting lubricants, artificial tears, ocular lubricants, ointments, emollients, liposomal spray, or $\omega-3$ dietary supplements), continued use was permitted during the study. The dosage and frequency of use was assessed at each visit. Subjects were instructed not to start using any new OTC products during the study.

After receiving either the VTP treatment or the crossover VTP treatment, subjects were provided with instructions (Figure S1) on how to perform daily blinking exercises 10 times a day for 1 month post-treatment along with tips for how to incorporate the exercises into their day. The actual frequency of blinking exercises performed was assessed at the 1 month post-treatment visit.

\section{Study groups}

For analysis purposes, there were two study groups: 1) the intent-to-treat (ITT) population (all randomized subjects) and 2) the per protocol (PP) population (all subjects who completed the study with no protocol deviations significantly affecting the integrity of the data). Safety analyses were performed with the ITT population. The primary and secondary endpoint analyses and the additional exploratory analyses were performed with both the ITT and the PP populations.

\section{Results}

There were no statistically significant $(\mathrm{p}>0.05)$ differences between groups or protocol revisions in demographics or baseline measurements of MGS score, SPEED score, LWE, LIPCOF, TBUT, or frequency in OTC drop use between the treatment and untreated control groups, supporting that subjects can be pooled across protocol revisions for these analyses. Furthermore, for the sub-study subjects, there was no statistically significant $(p>0.05)$ difference in the baseline degree of meibomian gland atrophy (meiboscore) between groups. Although there was a statistically significant difference $(\mathrm{p}<0.05)$ between groups in baseline total contact lens wear time, there was no significant $(\mathrm{p}>0.05)$ difference between groups in baseline comfortable contact lens wear time. As expected because of the difference in inclusion criteria between protocol revisions, there was a statistically significant $(\mathrm{p}<0.05)$ difference between revisions in baseline total and comfortable contact lens wear times. To control for these significant baseline differences, the exploratory contact lens wearing time analyses were stratified by protocol revisions (1 and 2) and groups.

Table 1 contains the demographics for the study subjects. The retention of subjects over the study duration was excellent. Subject accountability was $100 \%$ at 1 month, $98 \%$ at 3 months, and $96 \%$ at 4 months. One untreated control subject was discontinued prior to the 3-month visit because they were unable to complete the follow-up visit schedule.

\section{Primary and secondary effectiveness endpoints}

Table 2 shows the mean MGS and SPEED scores for the treatment and untreated control groups at baseline and 3 months. Between baseline and 3 months, the treatment group had a statistically significant greater mean change (improvement) in MGS score $(\mathrm{p}<0.0001)$, the number of functional meibomian glands (Table $3, p<0.0001$ ), and SPEED score $(\mathrm{p}<0.0001)$ compared to the untreated control group. Similar improvements were observed in the crossover treatment group assessed 1 month post-VTP treatment.

\section{Additional exploratory analyses \\ Comfortable contact lens wearing time}

Contact lens wear analyses were stratified by protocol revisions and groups because of the difference in inclusion criteria for contact lens wear time and to control associated statistically significant baseline differences in CL wear times.

Table I Demographics for treatment (VTP) and untreated control groups: ITT population

\begin{tabular}{llll}
\hline Group & $\begin{array}{l}\text { Treatment (VTP) } \\
(\mathbf{n = 2 9 )}\end{array}$ & $\begin{array}{l}\text { Untreated control } \\
(\mathbf{n = 2 6 )}\end{array}$ & $\begin{array}{l}\text { All subjects } \\
(\mathbf{n}=\mathbf{5 5})\end{array}$ \\
\hline $\begin{array}{l}\text { Age (years), mean } \pm \text { SD } \\
\text { Gender }\end{array}$ & $40.0 \pm 13.1$ & $43.5 \pm 15.9$ & $41.7 \pm 14.5$ \\
$\quad$ \% male & 13.8 & 15.4 & 14.6 \\
$\quad$ \% female & 86.2 & 84.6 & 85.4 \\
Race & & & 21.8 \\
$\quad$ \% Asian & 20.7 & 23.1 & 13.4 \\
\% Black/African American & 17.2 & 15.4 & 60.0 \\
\% White/Caucasian & 58.6 & 61.5 & 1.8 \\
\hline other race (multiracial) & 3.4 & 0.0 & \\
\hline
\end{tabular}

Abbreviations: ITT, intent-to-treat; SD, standard deviation; VTP, vectored thermal pulsation. 
Table 2 Summary of primary and secondary endpoints: ITT population

\begin{tabular}{|c|c|c|c|c|c|c|c|}
\hline \multirow{2}{*}{$\frac{\text { Group }}{\text { Visit }}$} & \multicolumn{3}{|c|}{ Treatment (VTP) } & \multicolumn{2}{|c|}{ Untreated control } & \multirow{2}{*}{$\begin{array}{l}\text { VTP vs control } \\
\text { Change baseline } \\
\text { to } 3 \text { months } \text { mo }^{2}\end{array}$} & \multirow{2}{*}{$\frac{\text { Crossover VTP }}{4 \text { months }}$} \\
\hline & Baseline & I month & 3 months & Baseline & 3 months & & \\
\hline Number $(n)$ of subjects & 29 & 29 & 29 & 26 & 25 & & 25 \\
\hline $\begin{array}{l}\text { Meibomian gland score } \\
(0-45) \text {, mean (SD) }\end{array}$ & $8.0(3.5)$ & $19.7(9.2)$ & $20.4(9.1)$ & $8.2(4.2)$ & $9.6(5.7)$ & $\mathrm{P}<0.000 \mathrm{I}$ & $22.4(9.4)$ \\
\hline $\begin{array}{l}\text { SPEED score (0-28), } \\
\text { mean (SD) }\end{array}$ & I4.5 (4.8) & $6.2(4.2)$ & $6.1(4.6)$ & I5.3 (4.5) & I4.5 (5.3) & $\mathrm{P}<0.000 \mathrm{I}$ & $7.4(5.0)^{*}$ \\
\hline
\end{tabular}

Notes: ${ }^{*} \mathrm{p}$-value based on two sample $t$-test; $\mathrm{p}<0.05$ statistically significant. *One crossover treatment subject did not complete all questions on SPEED questionnaire at 4 months ( $\mathrm{N}=24$ subjects in ITT population).

Abbreviations: ITT, intent-to-treat; SD, standard deviation; SPEED, Standard Patient Evaluation of Eye Dryness; VTP, vectored thermal pulsation.

Contact lens wear analyses by groups and protocol revisions are summarized in Table 4 and Figure 2.

On average, subjects wore contact lenses $6.4 \pm 1.0$ days per week in both the treatment and untreated control groups at baseline, which remained consistent at 3 months. Although the mean baseline total contact lens wear time per day was significantly longer for the treatment group than for the untreated control group (12.0 vs 10.2 hours, respectively; $\mathrm{p}=0.038$ ), there was no significant mean change in total wear time at 3 months for either group. Furthermore, there was no statistically significant difference $(p>0.05)$ in the mean change in total wear time from baseline to 3 months between groups overall or within each protocol revision.

However, the treatment group had a statistically significant greater mean increase from baseline to 3 months in the comfortable contact lens wear time than the untreated control group overall ( $3.7 \mathrm{vs}-0.3$ hours; $\mathrm{p}<0.0001$ ) for Protocol Revision 1 ( 4.3 vs -0.1 hours; $\mathrm{p}=0.005$ ) and Protocol Revision 2 ( 3.5 vs -0.3 hours; $p=0.0002$ ). In addition, the percentage of comfortable wear time out of total wear time per day was analyzed to control the baseline difference in total wear time between groups. From baseline to 3 months, the treatment group had a mean increase in the percentage of time contact lenses felt comfortable out of the total time worn per day from $45.0 \%( \pm 22.0 \%)$ at baseline to $74.4 \%$ $( \pm 23.4 \%)$ at 3 months. The mean change in the percentage was significantly greater for the treatment group as compared to the untreated control group overall (29.4 vs $-2.1 \%$, $\mathrm{p}<0.0001$ ) for Protocol Revision 1 (33.2 vs $-4.0 \%, \mathrm{p}=0.008$ ) and Protocol Revision 2 (28.1 vs $-1.4 \%, \mathrm{p}=0.0002$ ).

Table 3 Summary of statistically significant additional exploratory analyses

\begin{tabular}{|c|c|c|c|c|c|c|c|}
\hline \multirow{2}{*}{$\begin{array}{l}\text { Group } \\
\text { Visit }\end{array}$} & \multicolumn{3}{|c|}{ Treatment (VTP) } & \multicolumn{2}{|c|}{ Untreated control } & \multirow{2}{*}{$\begin{array}{l}\text { VTP vs control } \\
\text { Change baseline } \\
\text { to } 3 \text { months }\end{array}$} & \multirow{2}{*}{$\frac{\text { Crossover VTP }}{4 \text { months }}$} \\
\hline & Baseline & I month & 3 months & Baseline & 3 months & & \\
\hline $\begin{array}{l}\text { ITT population: number (n) } \\
\text { of subjects }\end{array}$ & 29 & 29 & 29 & 26 & 25 & & 25 \\
\hline $\begin{array}{l}\text { Topical OTC use (times/month), } \\
\text { mean (SD) }\end{array}$ & $56.3(45.1)$ & $39.8(30.8)$ & $26.0(30.6)$ & $44.1(36.0)$ & $42.2(39.1)$ & $\mathrm{p}=0.02^{\#}$ & $26.6(25.5)$ \\
\hline $\begin{array}{l}\text { Total OSDI score }(0-100) \text {, mean } \\
\text { (SD) }\end{array}$ & $39.6(16.4)$ & I5.7 (I3.4) & I3.4 (I5.5) & $40.8(20.3)$ & $37.5(23.8)$ & $\mathrm{p}=\mathbf{0 . 0 0 0 2 ^ { \# }}$ & I3.8(II.2) \\
\hline $\begin{array}{l}\text { Number of functional meibomian } \\
\text { glands ( } 0-15 \text { glands), mean (SD) }\end{array}$ & $1.9(1.6)$ & $6.9(3.8)$ & $7.1(3.6)$ & $2.1(1.7)$ & $2.5(2.2)$ & $\mathbf{p}<0.000$ I $^{\#}$ & $7.9(3.6)$ \\
\hline $\begin{array}{l}\text { Tear break-up time } \\
(0-20 \text { seconds), mean (SD) }\end{array}$ & $4.8(2.7)$ & $5.7(3.5)$ & $6.5(4.0)$ & $4.6(2.0)$ & $4.3(1.7)$ & $\mathrm{p}=0.0005^{\#}$ & $5.3(2.4)$ \\
\hline $\begin{array}{l}\text { LWE severity score }(0-3) \text {, } \\
\text { mean (SD) }\end{array}$ & I.8 (I.2) & I.5 (I.I) & $\mathrm{I} .4(\mathrm{I} .0)$ & $1.7(1.2)$ & $1.8(\mathrm{I} . \mathrm{I})$ & $\mathrm{p}=\mathbf{0 . 0 4 ^ { \ddagger }}$ & I.3 (I.0) \\
\hline $\begin{array}{l}\text { PP population: number }(n) \\
\text { of subjects }\end{array}$ & 25 & 25 & 25 & 24 & 24 & & 24 \\
\hline $\begin{array}{l}\text { Total conjunctival stain grade } \\
(0-18), \text { mean }(S D)\end{array}$ & $3.5(3.0)$ & $3.8(3.2)$ & $3.4(3.3)$ & $4.3(3.6)$ & $5.6(4.5)$ & $\mathrm{p}=\mathbf{0 . 0 4 ^ { \ddagger }}$ & $3.6(3.7)$ \\
\hline $\begin{array}{l}\text { Temporal LIPCOF grade }(0-3) \text {, } \\
\text { mean (SD) }\end{array}$ & I.7 (I.3) & $1.3(1.3)$ & I.I (I.2) & $1.5(1.1)$ & $\mathrm{I} .4(\mathrm{I} .0)$ & $\mathrm{p}=\mathbf{0 . 0 4 ^ { \ddagger }}$ & I.I (I.0) \\
\hline
\end{tabular}

Notes: ${ }^{p} \mathrm{p}$-value based on two sample $t$-test. ${ }^{\ddagger} \mathrm{p}$-value based on Wilcoxon rank sum test; $\mathrm{p}<0.05$, statistically significant. Bold font indicates statistical significance. Abbreviations: ITT, intent-to-treat; LIPCOF, lid parallel conjunctival folds; LWE, lid wiper epitheliopathy; OSDI, Ocular Surface Disease Index Questionnaire; OTC, overthe-counter; PP, per protocol; SD, standard deviation; VTP, vectored thermal pulsation. 
Table 4 Summary of contact lens wear analyses by protocol revision: ITT population

\begin{tabular}{|c|c|c|c|c|c|c|c|}
\hline \multirow{2}{*}{$\frac{\text { Group }}{\text { Visit }}$} & \multicolumn{3}{|c|}{ Treatment (VTP) } & \multicolumn{2}{|c|}{ Untreated control } & \multirow{2}{*}{$\begin{array}{l}\text { VTP vs control } \\
\text { Change baseline } \\
\text { to } 3 \text { months }{ }^{\#}\end{array}$} & \multirow{2}{*}{$\frac{\text { Crossover VTP }}{4 \text { months }}$} \\
\hline & Baseline & I month & 3 months & Baseline & 3 months & & \\
\hline Protocol & Revision I & & & & & & \\
\hline Number $(n)$ of subjects & 7 & 7 & 7 & 8 & 7 & & 7 \\
\hline $\begin{array}{l}\text { Total CL wear time/day } \\
\text { (hours), mean (SD) }\end{array}$ & $10.9(1.6)$ & $12.6(2.3)$ & $12.3(1.7)$ & $8.8(2.5)$ & $8.7(3.7)$ & $\mathrm{p}=0 . \mathrm{I}$ & $9.9(3.2)$ \\
\hline $\begin{array}{l}\text { Comfortable CL wear time/ } \\
\text { day (hours), mean (SD) }\end{array}$ & $3.1(1.1)$ & $8.3(4.0)$ & $7.4(2.6)$ & $2.9(I . I)$ & $2.6(1.3)$ & $p=0.005$ & $7.0(3.0)$ \\
\hline $\begin{array}{l}\text { \% comfortable wear/total } \\
\text { CL wear time, mean (SD) }\end{array}$ & $29.9(11.5)$ & $67.6(30.5)$ & $63.1(26.4)$ & $35.5(16.3)$ & 30.9 (II.5) & $p=0.008$ & $71.2(19.5)$ \\
\hline Protocol & Revision 2 & & & & & & \\
\hline Number $(n)$ of subjects & 22 & 22 & 22 & 18 & 18 & & 18 \\
\hline $\begin{array}{l}\text { Total CL wear time/day } \\
\text { (hours), mean (SD) }\end{array}$ & $12.3(3.4)$ & $12.2(3.2)$ & $12.3(3.3)$ & $10.8(3.1)$ & $10.7(3.5)$ & $\mathrm{p}=0.8$ & $10.9(2.8)$ \\
\hline $\begin{array}{l}\text { Comfortable CL wear time/ } \\
\text { day (hours), mean (SD) }\end{array}$ & $6.2(3.5)$ & $9.8(3.9)$ & $9.7(4.0)$ & $5.0(3.5)$ & $4.7(2.9)$ & $\mathrm{p}=0.0002$ & $8.4(2.9)$ \\
\hline $\begin{array}{l}\% \text { comfortable wear/total } \\
\text { CL wear time, mean (SD) }\end{array}$ & $49.9(22.5)$ & $79.6(21.1)$ & $78.0(21.7)$ & $48.0(31.5)$ & $46.6(24.0)$ & $\mathrm{p}=0.0002$ & $76.9(16.5)$ \\
\hline
\end{tabular}

Notes: " $\mathrm{p}$-value based on two sample t-test; $\mathrm{p}<0.05$ statistically significant. Bold font indicates statistical significance.

Abbreviations: ITT, intent-to-treat; CL, contact lens; SD, standard deviation; VTP, vectored thermal pulsation.

\section{DMI}

At US sites only where DMI was used to assess gland structure, the mean baseline average eyelid meiboscore was $1.8( \pm 0.7)$ for the treatment group $(\mathrm{n}=18$ subjects $)$ and $1.6( \pm 0.8)$ for the untreated control group ( $n=19$ subjects), reflecting evidence of gland atrophy in both groups. There was no statistically significant difference $(\mathrm{p}>0.05)$ in the mean baseline average eyelid meiboscore between the treatment and untreated control groups. Furthermore, there was no statistically significant difference $(\mathrm{p}>0.05)$ in the average eyelid meiboscore between baseline and 1 month post-VTP treatment for all treated eyes. The mean baseline average eyelid meiboscore for all subjects was 1.67 (95\% CI 1.4-1.9, n=37 subjects) (refer Figure 3 for examples of images captured using DMI).

\section{TBUT}

The treatment group demonstrated a statistically significant greater mean change in TBUT from baseline to 3 months than in the untreated control group ( 1.7 vs -0.2 seconds, respectively; $\mathrm{p}=0.0005$ ). The crossover treatment group showed a similar mean increase in TBUT at 4 months as the treatment group at 1 month (Table 3 ).

\section{Ocular surface staining}

There was no statistically significant difference $(\mathrm{p}>0.05)$ between the treatment and untreated control groups in the change in total corneal staining grade from baseline to 3 months. The treatment group showed significantly $(\mathrm{p}=0.04)$ less increase in the total conjunctival staining grade from

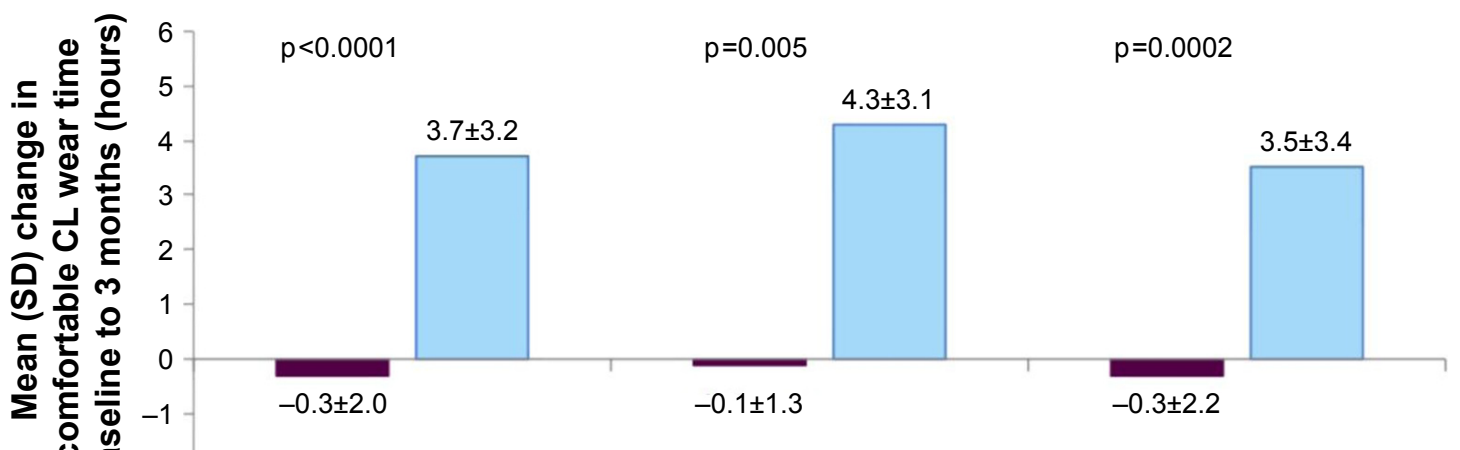

All subjects Protocol Revision 1

Protocol Revision 2

Untreated control $\quad \square$ LipiFlow $^{\circledast}$

Figure 2 The mean change in comfortable contact lens wear time from baseline to 3 months post-single VTP treatment. Abbreviations: $\mathrm{CL}$, contact lens; SD, standard deviation; VTP, vectored thermal pulsation. 

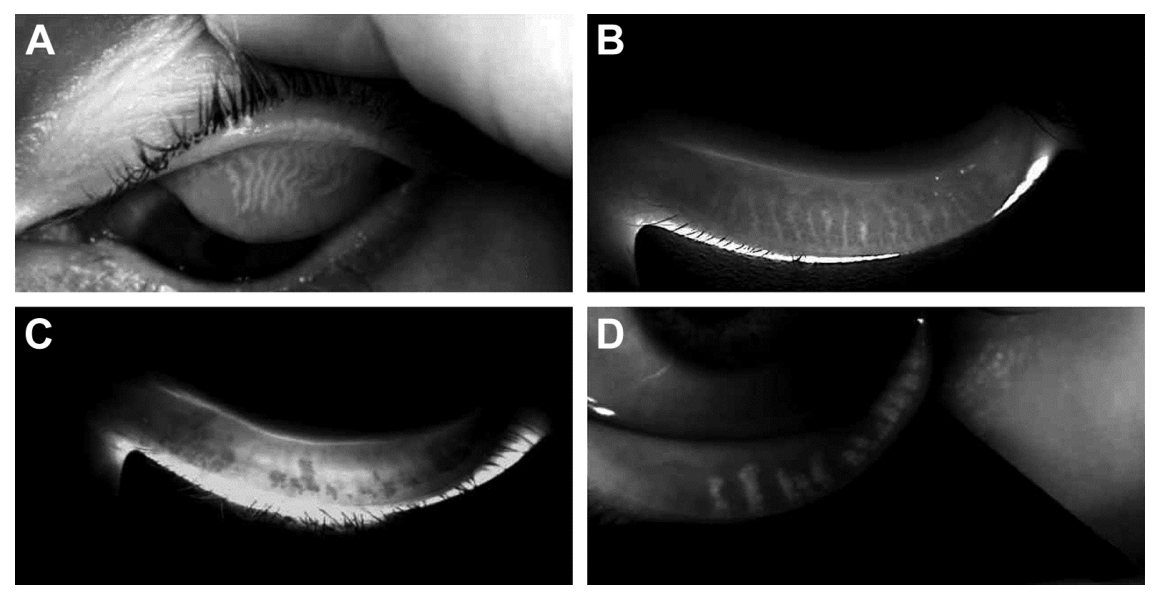

Figure 3 DMI images from four different study subjects.

Notes: The images indicate different degrees of gland atrophy and three different DMl illumination methods. (A) Upper left eyelid using reflective infra-red illumination. (B) Lower left eyelid using infra-red transillumination. (C) Lower left eyelid using infra-red transillumination. (D) Lower left eyelid using the merged reflective illumination and infra-red transillumination.

Abbreviation: DMI, Dynamic Meibomian Imaging.

baseline to 3 months than the untreated control group in the PP population (Table 3); however, the difference between groups was not statistically significant $(\mathrm{p}>0.05)$ in the ITT population.

\section{LWE}

The treatment group demonstrated statistically significantly $(p=0.048)$ greater reduction in the LWE severity grade from baseline to 3 months than the untreated control group (Table 3).

\section{Lid margin parallel conjunctival folds (LIPCOF)}

The treatment group had a statistically significantly $(\mathrm{p}=0.048)$ greater decrease in the temporal LIPCOF grade from baseline to 3 months than the untreated control group in the PP population (Table 3); however, the difference between groups was not significant $(\mathrm{p}>0.05)$ in the ITT population. For the nasal LIPCOF grade, there was no statistically significant $(p>0.05)$ difference in the change in grade from baseline to 3 months between groups.

\section{Frequency of OTC drop use}

At baseline, $69.0 \%$ of treatment subjects and $73.1 \%$ of untreated control subjects used at least one topical OTC medication; most of these subjects used only artificial tears. For only those subjects using topical OTC medications at baseline, $60.0 \%$ of the treatment group and $27.8 \%$ of the untreated control group had a decrease in the frequency of use from baseline to 3 months. The treatment group had a statistically significant greater mean decrease in the monthly frequency of topical OTC use from baseline to 3 months than the untreated control group ( $-30.3 \mathrm{vs}-3.8$ times per month; $\mathrm{p}=0.029)$ (Table 3 and Figure 4).

\section{Subject report of overall dry eye symptoms}

The vast majority of subjects reported an improvement in their overall dry eye symptoms: $93.1 \%$ of subjects at 1 month post-VTP and $86.2 \%$ of subjects at 3 months post-VTP in the treatment group reported this improvement and $100 \%$ of patients in crossover treatment group reported an improvement in their overall dry eye symptoms at 1 month post-VTP treatment. Furthermore, for those subjects who reported subjective improvement in overall dry eye symptoms after VTP treatment, the mean percentage improvement was $\sim 55 \%$ (specifically $56.3 \%$ at 1 month post-VTP and 59.6\% at 3 months post-VTP in the treatment group and $55.6 \%$ at 1 month post-VTP in the crossover treatment group).

\section{Subject report of improvement in tasks}

The vast majority of subjects reported an improvement in ability to perform a task better or longer, such as visual tasks (eg, reading, computer use, and driving) and wearing contact lenses longer or more comfortably: $82.8 \%$ of subjects at 1 month post-VTP and $72.4 \%$ of subjects at 3 months postVTP in the treatment group and $80.0 \%$ of subjects at 1 month post-VTP treatment in the crossover treatment group.

\section{Other questionnaire metrics}

All subjects had a dry eye diagnosis based on the CLDEQ at baseline. The treatment group had a statistically significant higher percentage of subjects who no longer had a dry eye diagnosis at 3 months based on the CLDEQ as compared 


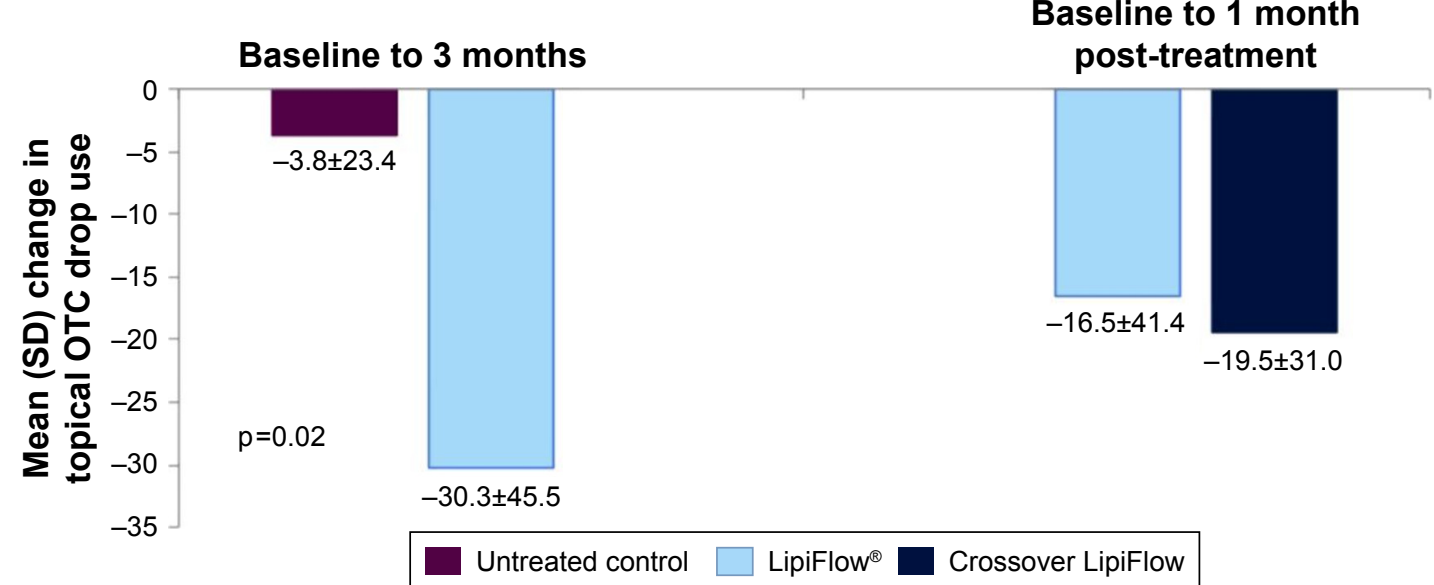

Figure 4 The mean change in OTC drop use frequency from baseline to 3 months post-single VTP treatment.

Abbreviations: OTC, over-the-counter; SD, standard deviation; VTP, vectored thermal pulsation.

to the untreated control group (44.8 vs $4.0 \%$, respectively; $\mathrm{p}=0.0006$ ).

The treatment group had a significantly greater reduction in dry eye symptoms than the untreated control group based on the mean change total OSDI score from baseline to 3 months ( -26.2 vs -2.6 , respectively; $\mathrm{p}=0.0002$ ). Correspondingly, the treatment group also demonstrated a statistically significant $(p<0.05)$ greater mean change from baseline to 3 months in the OSDI sub-scores for ocular symptoms, vision-related functioning, and environmental conditions than the untreated control group, as shown in Figure 5. The crossover treatment group displayed a similar reduction in symptoms based on the mean total OSDI score and sub-scores at 4 months and 1 month post-VTP treatment.

\section{Adverse events}

There were no adverse events reported as related to a device or a study procedure, and no serious adverse event or unanticipated adverse device effects were reported. These data validate the low-risk safety profile of the LipiFlow System, reported in prior studies. Eleven adverse events unrelated to a device were reported including five ocular events and six systemic events.

Furthermore, slit lamp findings observed immediately after VTP treatment in the treatment and crossover treatment groups, including eyelid edema, conjunctival edema, conjunctival hyperemia/injection, petechiae, and superficial punctate keratitis (SPK), were consistent with slit lamp findings noted post-treatment in prior studies. ${ }^{27-31}$ All immediate

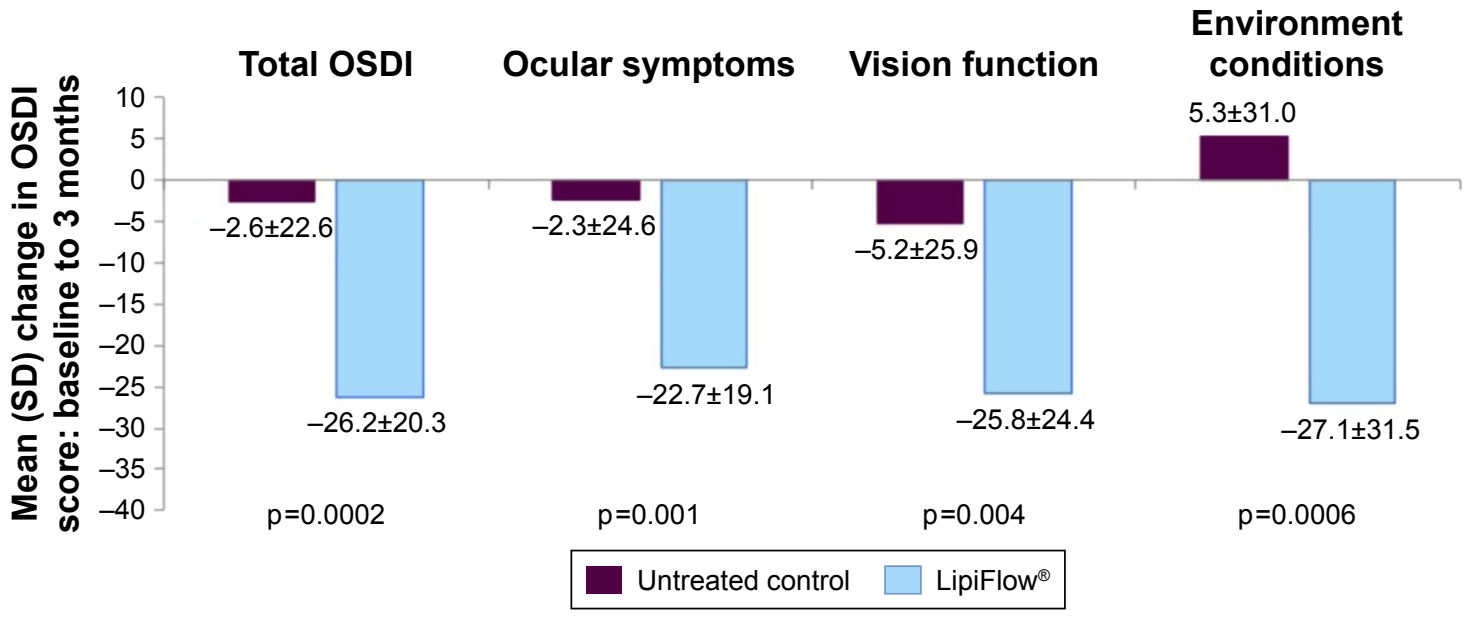

Figure 5 The mean change in OSDI total and component scores from baseline to 3 months post-single VTP treatment. Abbreviations: OSDI, Ocular Surface Disease Index; SD, standard deviation; VTP, vectored thermal pulsation. 
post-treatment slit lamp findings were transient and did not require medical treatment.

Changes in eyelid appearance and signs of obvious MGD after VTP treatment were not expected because MGD is a chronic condition resulting in long-standing morphological changes that can be permanent. However, a trend was observed in the treatment group for improvement from baseline to 3 months after VTP treatment with an increase from $41.4 \%$ to $58.6 \%$ of subjects with normal eyelid appearance and a decrease from $63.8 \%$ to $46.6 \%$ of subjects with signs of obvious MGD.

\section{Discussion}

The efficacy of a single VTP treatment in patients with MGD and dry eye is well established, ${ }^{27-33,49}$ and VTP treatment has been shown to deliver a sustained treatment effect in multiple studies. ${ }^{28,30,32,49}$ The majority of prior VTP studies have focused on treating MGD in dry eye populations, including Sjögren's patients ${ }^{32}$ and patients with refractory dry eye postrefractive surgery. ${ }^{49,50}$ However, contact lens wearers with MGD are a natural study population for the VTP treatment of MGD due to 1) the demands that a contact lens places on the tear film, ${ }^{1-13} 2$ ) the significant correlation of both severity and prevalence of MGD in contact lens wearers, ${ }^{11,16-20}$ and 3) the consistently high contact lens dropout rate due to dry eye symptoms. , $^{1,5,51-54}$

The primary and secondary endpoints in this study were met with the treatment group demonstrating a statistically significant greater mean improvement meibomian gland score and a mean reduction in SPEED score from baseline to 3 months compared to the untreated control group. Similar mean improvements in meibomian gland and SPEED scores were observed at 1 month after crossover VTP treatment of the control group, which further supports the effectiveness of the device. These study findings are consistent with the effectiveness results of multiple prior studies using the LipiFlow System for the treatment of MGD. On average, prior studies have shown that a single VTP treatment increases gland function approximately threefold and reduces dry eye symptoms by $\sim 50 \% .{ }^{28}$ In this study, measured 3 months postVTP treatment, the mean meibomian gland function score had increased by 2.6 times from baseline and the mean dry eye symptom score decreased by $58 \%$.

With regard to the imaging analysis of meibomian gland structure using a novel technology known as DMI, our data are consistent with the findings of prior experts. ${ }^{41}$ Arita et al reported an average meiboscore for SCL wearers as 1.52 (95\% CI 1.17-1.86, $\mathrm{n}=121$ subjects) compared to the average meiboscore of noncontact lens wearers, which was 0.48 (95\% CI 0.37-0.59, n=137 subjects). ${ }^{39,41}$ Our data showed an average meiboscore for contact lens wearers as $1.67(95 \%$ CI 1.4-1.9, $\mathrm{n}=37$ subjects).

The additional exploratory analyses reveal other benefits of VTP treatment for contact lens wearers with MGD. The treatment group showed statistically significantly greater mean improvements in the following parameters from baseline to 3 months as compared to the untreated control group: percentage of comfortable contact lens wear time out of the total contact lens wear time per day; monthly frequency of topical OTC medication use for MGD or dry eye; total OSDI score and sub-scores for ocular symptoms, vision-related functioning, and environmental conditions; percentage of subjects who no longer had a dry eye diagnosis based on the CLDEQ; number of functional meibomian glands; TBUT; and LWE severity. Furthermore, these findings were substantiated by similar mean improvements in the above parameters 1 month after crossover VTP treatment of the untreated control group.

This prospective, randomized, multicenter pilot clinical trial demonstrates the benefits of treating MGD in contact lens wearers. These results validate that a single VTP treatment can significantly improve meibomian gland function and tear film stability, decrease dependence on OTC medications, and reduce known contact lens-related ocular surface indicators of dryness and inflammation, such as LWE as early as 1 month following treatment. The most significant new finding in this study is that mean comfortable contact lens wear time increased, on average, by 4 hours per day at 1 month after VTP treatment, and the findings persisted over the entire study, approximately doubling the mean comfortable contact lens wearing time measured at baseline. This study also confirms that a single VTP treatment results in tangible improvement in the quality of life for most subjects, as evidenced by being able to perform a patient-reported task (such as using computers or digital devices, reading, and wearing contact lenses) significantly better or longer after treatment.

The timing of re-treatment was not addressed in this study. Prior studies on the use of VTP for MGD in noncontact lens wearing dry eye patients indicate that the VTP treatment effect for gland function and dry eye symptoms can be sustained for up to $\geq 12$ months. ${ }^{29,31}$ These studies also highlight that early intervention optimizes treatment efficacy $^{29}$ and that patients with more severe disease are likely to benefit from a combination of in-office treatment to address gland obstruction along with patient-specific at-home 
adjunctive therapies. ${ }^{31}$ How these findings relate to contact lens wearers with MGD remains to be tested.

The absence of device-related adverse events in this study further confirms the low-risk safety profile of the LipiFlow System, as demonstrated in prior studies. In addition, slit lamp findings observed immediately after VTP treatment were transient, required no medical treatment, and were consistent with prior studies.

While changes in eyelid appearance and signs of obvious MGD after VTP treatment were not expected because of chronic, long-standing nature of these morphological changes, improvement in eyelid appearance from baseline to 3 months after VTP treatment was observed and noted. These findings suggest that MGD-related eyelid and gland orifice changes can be improved through clearing of gland obstruction with the LipiFlow System.

The importance of blinking as a core mechanism for maintaining meibomian gland health is known; ${ }^{37,55-57}$ however, there is no accepted standard for how to communicate this to patients in a clinical setting. In this study, all subjects were provided very specific blinking instructions and told to perform them daily for a month after VTP treatment. Compliance with blinking was assessed at the 1 month posttreatment visit and more than half of the subjects reported completing 10 repetitions of the exercises 5-10 times a day for 20-30 days of the month. However, compliance with blinking exercises was not a focus of this study and documentation of daily compliance with blinking exercises was not included in the study protocol. The impact of blinking exercises on the efficacy of VTP treatment has not been directly tested. This relationship should be investigated in future studies.

There are limitations to every study, and this study is no exception. This was a small study intended to assess the value of performing a larger clinical study in contact lens wearing patients with MGD. The study was open label, and the control group was untreated. We cannot rule out investigator bias or the placebo effect. However, the safety and efficacy of the treatment have been well documented in both randomized controlled multicenter trials and retrospective studies and case series analyses over several years..$^{27-33,49,50}$

The purpose of this study was to assess the benefit of treating MGD in a contact lens wearing population with a single VTP treatment. The results clearly indicate that identifying and treating MGD in contact lens wearers have the potential to significantly improve meibomian gland function, reduce dry eye signs and symptoms, and dramatically increase comfortable contact lens wearing time. The data strongly support a prior conclusion that contact lens wearers should be routinely evaluated and treated for MGD. ${ }^{18}$

\section{Conclusion}

The study results establish that there are multiple benefits of VTP treatment for MGD in contact lens wearers with discomfort and that these benefits far outweigh the risks of the VTP device. Of particular interest is that a single VTP treatment increased mean comfortable wearing time by 4 hours, on average, approximately doubling the number of comfortable contact lens wearing hours. This result was sustained out to 3 months post-treatment on average.

\section{Acknowledgment}

This study was supported by TearScience Inc., Morrisville, NC, USA.

\section{Disclosure}

$\mathrm{CAB}$ and $\mathrm{CAC}$ are employees of TearScience, Inc. The other authors report no conflicts of interest in this work.

\section{References}

1. Young G. Why one million contact lens wearers dropped out. Cont Lens Anterior Eye. 2004;27(2):83-85.

2. Young G, Chalmers R, Napier L, Kern J, Hunt C, Dumbleton K. Soft contact lens-related dryness with and without clinical signs. Optom Vis Sci. 2012;89(8):1125-1132.

3. Caffery B, Dogru M, Jones LW, et al. Contact lens comfort. Optom Vis Sci. 2016;93(8):790-792.

4. Rah MJ, Merchea MM, Doktor MQ. Reducing dropout of contact lens wear with Biotrue multipurpose solution. Clin Ophthalmol. 2014;8: 293-299.

5. Rumpakis J [webpage on the Internet]. New data on contact lens dropouts: an international perspective. Review of Optometry; 2010. Available from: http://www.revoptom.com/content/d/contact_lenses/ i/990/c/18929/. Accessed October 2, 2017.

6. Craig JP, Willcox MD, Argüeso P, et al. The TFOS International Workshop on Contact Lens Discomfort: report of the contact lens interactions with the tear film subcommittee. Invest Ophthalmol Vis Sci. 2013; 54(11):TFOS123-TFOS156.

7. Jones L, Brennan NA, González-Méijome J, et al. The TFOS International Workshop on Contact Lens Discomfort: report of the contact lens materials, design, and care subcommittee. Invest Ophthalmol Vis Sci. 2013;54(11):TFOS37-TFOS70.

8. Navascues-Cornago M, Morgan PB, Maldonado-Codina C. Effect of Three Interventions on Contact Lens Comfort in symptomatic wearers: a randomized clinical trial. PLoS One. 2015;10(8):e0135323.

9. Korb DR, Greiner JV, Herman JP, et al. Lid-wiper epitheliopathy and dry-eye symptoms in contact lens wearers. CLAO J. 2002;28(4): 211-216.

10. Pult H, Purslow C, Berry M, Murphy PJ. Clinical tests for successful contact lens wear: relationship and predictive potential. Optom Vis Sci. 2008;85(10):E924-E929.

11. Arita R, Fukuoka S, Morishige N. Meibomian gland dysfunction and contact lens discomfort. Eye Contact Lens. 2017;43(1):17-22.

12. Efron N, Jones L, Bron AJ, et al. The TFOS International Workshop on Contact Lens Discomfort: report of the contact lens interactions with the ocular surface and adnexa subcommittee. Invest Ophthalmol Vis Sci. 2013;54:TFOS98Y122. 
13. Craig JP, Nichols KK, Akpek EK, et al. TFOS DEWS II definition and classification report. Ocul Surf. 2017;15(3):276-283.

14. Nichols KK, Redfern RL, Jacob JT, et al. The TFOS International Workshop on Contact Lens Discomfort: report of the definition and classification subcommittee. Invest Ophthalmol Vis Sci. 2013;54(11): TFOS14-TFOS19.

15. Suhalim JL, Parfitt GJ, Xie Y, et al. Effect of desiccating stress on mouse meibomian gland function. Ocul Surf. 2014;12(1):59-68.

16. Henriquez AS, Korb DR. Meibomian glands and contact lens wear. Br J Ophthalmol. 1981;65(2):108-111.

17. Paugh JR, Knapp LL, Martinson JR, Hom MM. Meibomian therapy in problematic contact lens wear. Optom Vis Sci. 1990;67(11):803-806.

18. Machalińska A, Zakrzewska A, Adamek B, et al. Comparison of morphological and functional meibomian gland characteristics between daily contact lens wearers and nonwearers. Cornea. 2015;34(9): 1098-1104.

19. Cox SM, Berntsen DA, Chatterjee N, et al; Performance of Contact Lens Solutions Study Group. Eyelid margin and meibomian gland characteristics and symptoms in lens wearers. Optom Vis Sci. 2016;93(8): 901-908.

20. Alghamdi WM, Markoulli M, Holden BA, Papas EB. Impact of duration of contact lens wear on the structure and function of the meibomian glands. Ophthalmic Physiol Opt. 2016;36(2):120-131.

21. Ong BL. Relation between contact lens wear and meibomian gland dysfunction. Optom Vis Sci. 1996;73:208-210.

22. Mudgil P. Antimicrobial role of human meibomian lipids at the ocular surface. Invest Ophthalmol Vis Sci. 2014;55(11):7272-7277.

23. Azizi S, Uçak T, Yaşar I, Karakurt Y, Erdogan E, Salman I. Evaluation of the corneal layers in meibomian-gland-dysfunction-related dry eye by in vivo slit-scanning confocal microscopy. Semin Ophthalmol. 2017;32(3):377-383.

24. Liang Q, Pan Z, Zhou M, et al. Evaluation of optical coherence tomography meibography in patients with obstructive meibomian gland dysfunction. Cornea. 2015;34(10):1193-1199.

25. Baudouin C, Messmer EM, Aragona P, et al. Revisiting the vicious circle of dry eye disease: a focus on the pathophysiology of meibomian gland dysfunction. Br J Ophthalmol. 2016;100(3):300-306.

26. Korb DR, Blackie CA. A single LipiFlow treatment increases soft contact lens wearing time and reduces lid wiper epitheliopathy and dry eye symptoms. Abstract presented at the AAO; Denver, CO; 2014.

27. Lane SS, DuBiner HB, Epstein RJ, et al. A new system, the LipiFlow, for the treatment of meibomian gland dysfunction. Cornea. 2012;31(4): 396-404.

28. Blackie CA, Carlson AN, Korb DR. Treatment for meibomian gland dysfunction and dry eye symptoms with a single-dose vectored thermal pulsation: a review. Curr Opin Ophthalmol. 2015;26(4):306-313.

29. Blackie CA, Coleman CA, Holland EJ. The sustained effect (12 months) of a single-dose vectored thermal pulsation procedure for meibomian gland dysfunction and evaporative dry eye. Clin Ophthalmol. 2016;10: $1385-1396$

30. Friedland BR, Fleming CP, Blackie CA, Korb DR. A novel thermodynamic treatment for meibomian gland dysfunction. Curr Eye Res. 2011; 36(2):79-87.

31. Greiner JV. Long-term ( 3 year) effects of a single thermal pulsation system treatment on meibomian gland function and dry eye symptoms. Eye Contact Lens. 2016;42(2):99-107.

32. Epitropoulos AT, Goslin K, Beda R, Blackie CA. Meibomian gland dysfunction patients with novel Sjögren's syndrome biomarkers benefit significantly from a single vectored thermal pulsation procedure: a retrospective analysis. Clin Ophthalmol. 2017;11:701-706.

33. Finis D, König C, Hayajneh J, Borrelli M, Schrader S, Geerling G. Sixmonth effects of a thermodynamic treatment for MGD and implications of meibomian gland atrophy. Cornea. 2014;33(12):1265-1270.

34. Ngo W, Situ P, Keir N, Korb D, Blackie CA, Simpson T. Psychometric properties and validation of the Standard Patient Evaluation of Eye Dryness questionnaire. Cornea. 2013;32(9):1204-1210.
35. Nichols JJ, Mitchell GL, Nichols KK, Chalmers R, Begley C. The performance of the contact lens dry eye questionnaire as a screening survey for contact lens-related dry eye. Cornea. 2002;21(5):469-475.

36. Korb DR, Blackie CA. Debridement-scaling: a new procedure that increases meibomian gland function and reduces dry eye symptoms. Cornea. 2013;32(12):1554-1557.

37. Wan $T$, Jin X, Lin L, Xu Y, Zhao Y. Incomplete blinking may attribute to the development of meibomian gland dysfunction. Curr Eye Res. 2016; 41(2):179-185.

38. Korb DR, Blackie CA. Meibomian gland diagnostic expressibility: correlation with dry eye symptoms and gland location. Cornea. 2008; 27(10):1142-1147.

39. Arita R, Itoh K, Inoue K, Amano S. Noncontact infrared meibography to document age-related changes of the meibomian glands in a normal population. Ophthalmology. 2008;115(5):911-915.

40. Arita R, Itoh K, Maeda S, et al. Proposed diagnostic criteria for obstructive meibomian gland dysfunction. Ophthalmology. 2009;116(11): 2058.e1-2063.e1.

41. Arita R, Itoh K, Inoue K, Kuchiba A, Yamaguchi T, Amano S. Contact lens wear is associated with decrease of meibomian glands. Ophthalmology. 2009;116(3):379-384.

42. Korb DR, Greiner JV, Herman J. Comparison of fluorescein break-up time measurement reproducibility using standard fluorescein strips versus the Dry Eye Test (DET) method. Cornea. 2001;20(8):811-815.

43. Lemp MA. Report of the National Eye Institute/Industry workshop on Clinical Trials in Dry Eyes. CLAO J. 1995;21(4):221-232.

44. Nichols KK, Morris S, Gaddie IB, Evans D. Epinastine $0.05 \%$ ophthalmic solution in contact lens-wearing subjects with a history of allergic conjunctivitis. Eye Contact Lens. 2009;35(1):26-31.

45. Nichols JJ, Bickle KM, Zink RC, Schiewe MD, Haque RM, Nichols KK. Safety and efficacy of topical azithromycin ophthalmic solution $1.0 \%$ in the treatment of contact lens-related dry eye. Eye Contact Lens. 2012;38(2):73-79.

46. Nichols JJ, Mitchell GL, Nichols KK. An assessment of self-reported disease classification in epidemiological studies of dry eye. Invest Ophthalmol Vis Sci. 2004;45(10):3453-3457.

47. Schiffman RM, Christianson MD, Jacobsen G, Hirsch JD, Reis BL. Reliability and validity of the ocular surface disease index. Arch Ophthalmol. 2000;118(5):615-621.

48. Miller KL, Walt JG, Mink DR, et al. Minimal clinically important difference for the ocular surface disease index. Arch Ophthalmol. 2010; 128(1):94-101.

49. Schallhorn CS, Schallhorn JM, Hannan S, Schallhorn SC. Effectiveness of an eyelid thermal pulsation procedure to treat recalcitrant dry eye symptoms after laser vision correction. J Refract Surg. 2017;33(1):30-36.

50. Petzold G, Bedi R, Blackie CA. Management of dry-eye syndrome after laser in situ keratomileusis with a vectored thermal pulsation system. JCRS Online Case Rep. 2016;4:34-37.

51. Berberian D. The contact lens 'drop-out' - a dissatisfied person? Ophthalmic Optician. 1983;17:551.

52. Fonn D. Targeting contact lens induced dryness and discomfort: what properties will make lenses more comfortable. Optom Vis Sci. 2007;84(4):279-285.

53. Pritchard N, Fonn D, Brazeau D. Discontinuation of contact lens wear: a survey. Int Contact Lens Clin. 1999;26(6):157-162.

54. Richdale K, Sinnott LT, Skadahl E, Nichols JJ. Frequency of and factors associated with contact lens dissatisfaction and discontinuation. Cornea. 2007;26(2):168-174.

55. Linton RG, Curnow DH, Riley WJ. The meibomian glands. An investigation into the secretion and some aspects of the physiology. $\mathrm{Br} J$ Ophthalmol. 1961;45:718-723.

56. McMonnies CW. Incomplete blinking: exposure keratopathy, lid wiper epitheliopathy, dry eye, refractive surgery, and dry contact lenses. Cont Lens Anterior Eye. 2007;30(1):37-51.

57. McMonnies CW. Blink efficiency: a neglected area of ocular surface disease management? Invest Ophthalmol Vis Sci. 2011;52(7):4484. 


\section{Supplementary material}

1. Hold your fingers at the corners of your eyes

to feel the lid movement.

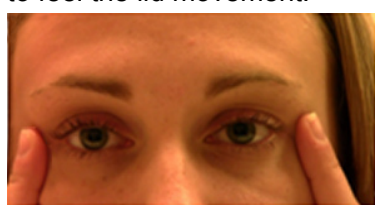

2. Close your eyelids together with the upper and lower lids completely touching for a count of 2. When closing your lids correctly, you will feel no lid movement under your fingers.

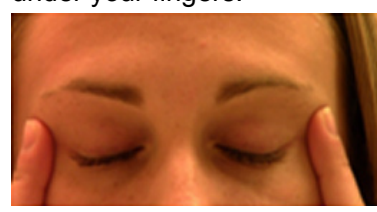

3. Squeeze your eyelids lightly for a count of 2 . When squeezing your eyelids lightly, you will feel slight lid movement under your fingers. With a moderate squeeze, you should feel the contraction of the lid muscles under your fingers.

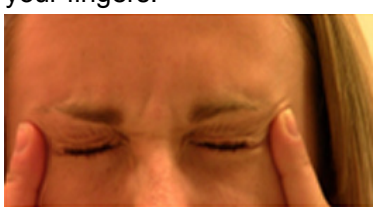

4. Open your eyelids to complete the blinking cycle for a count of 2 .

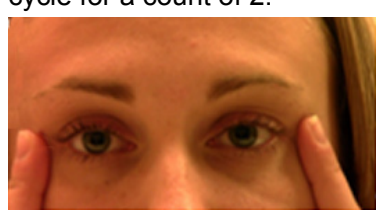

5. Repeat the following sequence nine more times for a total of 10 repetitions: close and count 1 and 2 , squeeze and count 1 and 2 , and open and count 1 and 2 .

Figure SI Blinking exercises.

Note: Perform 10 repetitions every hour for a minimum of 10 times per day every day.

\section{Publish your work in this journal}

Clinical Ophthalmology is an international, peer-reviewed journal covering all subspecialties within ophthalmology. Key topics include: Optometry; Visual science; Pharmacology and drug therapy in eye diseases; Basic Sciences; Primary and Secondary eye care; Patient Safety and Quality of Care Improvements. This journal is indexed on

Submit your manuscript here: http://www.dovepress.com/clinical-ophthalmology-journal

\section{Dovepress}

PubMed Central and CAS, and is the official journal of The Society of Clinical Ophthalmology (SCO). The manuscript management system is completely online and includes a very quick and fair peer-review system, which is all easy to use. Visit http://www.dovepress.com/ testimonials.php to read real quotes from published authors. 